\title{
Correlation between Helicobacter pylori Infection and Metabolic Abnormality in General Population: A Cross-Sectional Study
}

\author{
Li-juan Lu, ${ }^{1,2}$ Ning-Bo Hao, ${ }^{1}$ Jian-Jun Liu, ${ }^{1}$ Xue Li, ${ }^{1}$ and Rui-Ling Wang $\mathbb{D}^{1}$ \\ ${ }^{1}$ Department of Gastroenterology, General Hospital of the PLA Rocket Force, Beijing, China \\ ${ }^{2}$ Jinzhou Medical University, Jinzhou, China \\ Correspondence should be addressed to Rui-Ling Wang; wang.rui.lin.g@163.com
}

Received 28 October 2017; Revised 1 February 2018; Accepted 7 February 2018; Published 20 March 2018

Academic Editor: Tatsuya Toyokawa

Copyright (c) $2018 \mathrm{Li}$-juan Lu et al. This is an open access article distributed under the Creative Commons Attribution License, which permits unrestricted use, distribution, and reproduction in any medium, provided the original work is properly cited.

\begin{abstract}
Background. Previous studies have suggested a link between Helicobacter pylori (H. pylori) and metabolic abnormality. This study aimed at investigating the correlation between $H$. pylori infection and metabolic abnormality in a general population. Methods. All enrolled participants underwent a carbon-13 urea breath test $\left({ }^{13} \mathrm{C}-\mathrm{UBT}\right)$. For each individual, the following data were collected: age, gender, alanine transaminase (ALT), total protein, albumin, cholesterol, triglyceride (TG), high-density lipoprotein cholesterol (HDL-C), low-density lipoprotein cholesterol (LDL-C), urea nitrogen, creatinine, uric acid, fasting plasma glucose, postprandial blood sugar, nonalcoholic fatty liver disease (NAFLD), and bone mineral density (BMD). Results. The study included 1867 (393 females and 1474 males, aged $54.0 \pm 9.6$ years) people that took a physical examination. There was no significant difference in gender and age between the study participants with and without $H$. pylori infection. The statistical data are as follows: albumin: $P=0.045$, uric acid: $P=0.025$, fasting glucose: $P=0.043$, and postprandial blood glucose: $P=0.035$. In terms of the patients with NAFLD, there were significant differences in ALT and HDL-C between the study participants with and without $H$. pylori infection. TG $(P=0.048)$, HDL-C $(P=0.011)$, and fasting blood glucose $(P=0.018)$ were significantly different in both groups among individuals who got osteopenia. Conclusion. H. pylori infection may be an important factor affecting metabolic abnormality and osteoporosis.
\end{abstract}

\section{Introduction}

H. pylori, a Gram-negative, spiral-shaped bacterium that dwells on the gastric epithelium, has an influence on approximately $50 \%$ of the human population, with a high rate in those living in developing countries. The prevalence of $H$. pylori infection is approximately $30 \%$ in developed countries and up to $80 \%$ in developing countries [1]. H. pylori infection induces chronic inflammation and immune responses in the stomach. H. pylori infection increases inflammatory factors, such as interleukin 1 (IL-1), interleukin 8 (IL-8), and tumor necrosis factor-alpha (TNF- $\alpha$ ). These inflammatory factors may result in metabolic changes and systemic immune responses [2-4]. Moreover, $H$. pylori is the most prevalent pathogenic bacteria in the stomach and can lead to a wide array of gastric disorders, including chronic gastritis, peptic ulcer, gastric cancer, and gastric mucosa-associated lymphoid tissue lymphoma (MALT) [5]. It is also considered a class I carcinogen. In recent years, accumulating evidence has demonstrated the role of $H$. pylori infection in extragastrointestinal diseases, such as cardiovascular diseases, neurological disorders, metabolic diseases, and kidney disease [6-8]. IL-1 and TNF- $\alpha$ trigger bone resorption [9]. In addition, osteoporosis is a condition with progressively decreasing $\mathrm{BMD}$ and increased bone fragility, with increased risk of bone fractures [10]. Metabolic syndrome and osteoporosis have become worldwide public health issue, especially in elderly subjects, with huge clinical and economic burdens. Identifying risk factors with potential therapeutic implications is increasingly important in managing metabolic abnormality and osteoporosis and decreasing economic and health burdens.

This study aimed at analyzing the correlation between $H$. pylori infection and metabolic abnormality in middle-aged and the elderly general population. 


\section{Material and Method}

This study was conducted at the General Hospital of PLA Rocket Force. All qualified subjects who attended their annual health examination during the year 2016 were initially enrolled.

2.1. Ethics. All subjects signed an informed consent prior to their enrollment in the study. Additionally, the study was planned according to the ethical guidelines following the declaration of the General Hospital of PLA Rocket Force.

2.2. Inclusion and Exclusion Criteria. Inclusion criteria were complete, having not used proton pump inhibitors (PPIs), histamine type 2 receptor antagonists $\left(\mathrm{H}_{2} \mathrm{~A}\right)$, antibiotics, bismuth, or sucralfate for up to one month prior to the urea breath test.

Individuals who had received vitamin D supplementation, H. pylori eradication therapy, or antibiotics were excluded. Other exclusion criteria include self-reported history of chronic liver disease or cirrhosis, alcohol inta$\mathrm{ke} \geq 30 \mathrm{~g} /$ day for men and $20 \mathrm{~g} /$ day for women, and positive serologic markers for hepatitis B or C virus. Participants with missing data on important covariates were excluded.

2.3. Method. In total, 1867 subjects who met these criteria were selected. All subjects who participated in a physical examination, from May 2016 to July 2016, were asked to take blood tests, a BMD examination, abdominal ultrasonography (US), and complete ${ }^{13} \mathrm{C}$-UBT for the diagnosis of active $H$. pylori infection. Biochemical investigation including ALT, total protein, albumin, cholesterol, TG, HDL-C, LDL-C, urea nitrogen, creatinine, uric acid, fasting plasma glucose, and postprandial blood sugar was performed in all individuals. After a $12 \mathrm{~h}$ overnight fast, $15 \mathrm{~mL}$ of blood was collected from the antecubital vein. It was analyzed within 4 hours after collection for biochemistry tests. After an overnight fast, ${ }^{13} \mathrm{C}$-UBT was performed using the Proto Pylori kit (Isodiagnostika Canada), containing $75 \mathrm{mg}$ of ${ }^{13} \mathrm{C}$-UBT and additives. Two breath samples were collected within a 30-minute interval. Patient samples were analyzed by gas chromatography. The results were expressed as delta over baseline (DOB). BMD was measured by a quantitative ultrasound test (QUS, Sahara Clinical Bone Sonometer, HOLOGIC, Bedford, MA, USA) method in the calcaneus in all participants. Fatty liver was examined using a Philips HD 11 XE multifunction color Doppler diagnostic instrument, according to the standard criteria for NAFLD by the Chinese Hepatology Association dating from February 2006 [1]. All ultrasound measurements were performed by the same ultrasound physician throughout the study. The diagnostic criteria are as follows: ALT $>40 \mathrm{U} / \mathrm{L}$, total protein $>5.69 \mathrm{mmol} / \mathrm{L}$, albumin $>55 \mathrm{~g} / \mathrm{L}$, cholesterol $>5.69$ $\mathrm{mmol} / \mathrm{L}, \mathrm{TG}>1.7 \mathrm{mmol} / \mathrm{L}, \mathrm{HDL}-\mathrm{C}>1.7 \mathrm{mmol} / \mathrm{L}, \mathrm{LDL}-\mathrm{C}<$ $3.64 \mathrm{mmol} / \mathrm{L}$, urea nitrogen $>8.05 \mathrm{mmol} / \mathrm{L}$, creatinine $>115$ $\mu \mathrm{mol} / \mathrm{L}$, uric acid $>416 \mu \mathrm{mol} / \mathrm{L}$, fasting plasma glucose $>$ $6.1 \mathrm{mmol} / \mathrm{L}$, and postprandial blood sugar $>7.8 \mathrm{mmol} / \mathrm{L}$. Because each year the same population undergo for a physical examination in our hospital, we had access to
TABLE 1: Characteristics of all participants.

\begin{tabular}{lc}
\hline Number (women/man) & $393 / 1474$ \\
Age & $54.0 \pm 9.6$ \\
ALT & $19.9 \pm 12.7$ \\
Total protein & $72.5 \pm 3.7$ \\
Albumin & $46.0 \pm 2.3$ \\
Cholesterol & $4.7 \pm 0.9$ \\
TG & $1.5 \pm 0.9$ \\
HDL-C & $1.3 \pm 0.4$ \\
LDL-C & $3.1 \pm 0.8$ \\
Urea nitrogen & $5.3 \pm 1.3$ \\
Creatinine & $79.0 \pm 15.1$ \\
Uric acid & $334.4 \pm 78.3$ \\
Fasting plasma glucose & $5.3 \pm 1.1$ \\
Postprandial blood sugar & $7.0 \pm 2.4$ \\
H. pylori positive & $589(31.5 \%)$ \\
NAFLD & $596(31.9 \%)$ \\
BMD & $900(48.2 \%)$ \\
\hline
\end{tabular}

information that could identify individual participants during or after data collection.

2.4. Statistical Analysis. Statistical analysis was performed using SPSS software version 17.0 (SPSS Inc., Chicago, IL). Continuous variables were checked by $t$-test, and the categorical variables were compared by a chi square test or Fisher exact probability method. Data are expressed as the mean and standard deviation. For all statistical analyses, the level of significance was considered to be $P<0.05$.

\section{Results}

A total of 1867 participants (393 women and 1474 men) were included in this study. The characteristics of all individuals are presented in Table 1 . The prevalence of $H$. pylori positivity was found to be $31.5 \%(589 / 1867)$. NAFLD was found to be $31.9 \%$ (596/1867). BMD deficiency was found in $48.2 \%$ of participants (900/1867).

The characteristics classified being $H$. pylori-positive or H. pylori-negative are shown in Table 2. It was observed that the individuals with high fasting plasma glucose had a higher rate $H$. pylori infection than those without $H$. pylori $(13.8 \%$ versus $8.5 \%, P=0.001)$. No difference was found between the two groups in terms of age or gender. In addition, fasting plasma glucose $(5.36 \pm 1.20$ versus $5.25 \pm 1.03, P=0.043)$ and postprandial blood sugar $(7.21 \pm 2.50$ versus $6.96 \pm 2.29$, $P=0.035)$ were significantly lower in the $H$. pylori-negative group, respectively.

In this study, participants with NAFLD had a higher rate of $H$. pylori infection than those without NAFLD (33.8\% versus $31.1 \%$ ). However, there were no statistically significant differences in NAFLD and $H$. pylori infection between the participants in this study $(P=0.241)$. Furthermore, we selected patients who were diagnosed with NAFLD. Those patients were divided into 2 groups according to $H$. pylori infection. Statistical results are shown in Table 3. The 
TABLE 2: Characteristics of categorical variables of all participants based on $H$. pylori infection.

\begin{tabular}{lccc}
\hline Variables & H. pylori $(+)$ & H. pylori $(-)$ & $P$ value \\
\hline Number (women/man) & $114 / 475$ & $279 / 999$ & 0.223 \\
BMD & $287(48.7 \%)$ & $613(48.0 \%)$ & 0.765 \\
NAFLD & $199(33.8 \%)$ & $397(31.1 \%)$ & 0.241 \\
ALT & $35(5.9 \%)$ & $58(4.5 \%)$ & 0.195 \\
Cholesterol & $74(12.6 \%)$ & $134(10.5 \%)$ & 0.185 \\
TG & $161(27.3 \%)$ & $314(24.6 \%)$ & 0.202 \\
HDL-C & $103(17.5 \%)$ & $194(15.2 \%)$ & 0.205 \\
LDL-C & $145(24.6 \%)$ & $320(25.0 \%)$ & 0.845 \\
Creatinine & $8(1.4 \%)$ & $14(1.1 \%)$ & 0.625 \\
Urea nitrogen & $20(3.4 \%)$ & $28(2.2 \%)$ & 0.126 \\
Uric acid & $99(16.8 \%)$ & $174(13.6 \%)$ & 0.070 \\
Fasting plasma glucose & $81(13.8 \%)$ & $109(8.5 \%)$ & $0.001^{*}$ \\
Postprandial blood sugar & $142(24.1 \%)$ & $287(22.5 \%)$ & 0.430 \\
\hline
\end{tabular}

※ represents $P$ value less than 0.05 between $H$. pylori-positive and $H$. pylorinegative groups.

TABLE 3: Characteristics of categorical variables of patients diagnosed as nonalcoholic fatty liver disease (NAFLD) on $H$. pylori infection.

\begin{tabular}{lccc}
\hline Variables & H. pylori $(+)$ & H. pylori $(-)$ & $P$ value \\
\hline Number (women/man) & $17 / 182$ & $65 / 332$ & $0.009^{*}$ \\
BMD & $111(56.9 \%)$ & $193(49.5 \%)$ & 0.090 \\
ALT & $19(9.5 \%)$ & $33(8.3 \%)$ & 0.926 \\
Cholesterol & $29(14.6 \%)$ & $51(12.8 \%)$ & 0.560 \\
TG & $94(47.2 \%)$ & $171(43.1 \%)$ & 0.335 \\
HDL-C & $68(34.2 \%)$ & $100(25.2 \%)$ & $0.022^{*}$ \\
LDL-C & $57(28.6 \%)$ & $110(27.7 \%)$ & 0.810 \\
Creatinine & $3(1.5 \%)$ & $7(1.8 \%)$ & 0.819 \\
Urea nitrogen & $4(2.0 \%)$ & $9(2.3 \%)$ & 0.840 \\
Uric acid & $49(24.6 \%)$ & $79(19.9 \%)$ & 0.185 \\
Fasting plasma glucose & $40(20.1 \%)$ & $64(16.1 \%)$ & 0.227 \\
Postprandial blood sugar & $63(31.7 \%)$ & $135(34.0 \%)$ & 0.566 \\
\hline
\end{tabular}

※ represents $P$ value less than 0.05 between $H$. pylori-positive and $H$. pylorinegative groups.

prevalence of NAFLD in the group with and without $H$. pylori were 199 patients (33.4\%) and 397 patients (66.6\%), respectively. Moreover, a significant difference was determined between the two groups in terms of gender $(P=0.009)$. Additionally, when $H$. pylori-positive and $H$. pylori-negative patients were compared, ALT $(26.04 \pm 15.68$ versus 23.37 $\pm 13.42, P=0.031$ ) levels were found to be significantly higher in $H$. pylori-positive patients; however, HDL-C $(1.12 \pm 0.26$ versus $1.19 \pm 0.28, P=0.005)$ levels were found to be lower with $H$. pylori infection.

Finally, the patients who were diagnosed with osteoporosis were picked up. Similarly, those patients were divided into 2 groups based on $H$. pylori infection. The results are shown in Table 4. We could clearly find that the prevalence of low HDL-C in the group with and without $H$. pylori was 54 patients $(18.8 \%)$ and 76 patients $(12.4 \%)$, respectively, in
TABLE 4: Characteristics of categorical variables of patients diagnosed as osteoporosis based on $\mathrm{H}$. pylori infection.

\begin{tabular}{lccc}
\hline Variables & H. pylori $(+)$ & H. pylori $(-)$ & $P$ value \\
\hline Number (women/man) & $57 / 230$ & $151 / 462$ & 0.119 \\
ALT & $20(7.0 \%)$ & $29(4.7 \%)$ & 0.168 \\
Cholesterol & $41(14.3 \%)$ & $67(10.9 \%)$ & 0.149 \\
TG & $82(28.6 \%)$ & $149(24.3 \%)$ & 0.172 \\
HDL-C & $54(18.8 \%)$ & $76(12.4 \%)$ & $0.011^{*}$ \\
LDL-C & $75(26.1 \%)$ & $166(27.1 \%)$ & 0.765 \\
Urea nitrogen & $14(4.9 \%)$ & $15(2.4 \%)$ & 0.054 \\
Creatinine & $5(1.7 \%)$ & $9(1.5 \%)$ & 0.757 \\
Uric acid & $44(15.3 \%)$ & $80(13.1 \%)$ & 0.355 \\
Fasting plasma glucose & $43(15.0 \%)$ & $59(9.6 \%)$ & $0.018^{*}$ \\
Postprandial blood sugar & $70(24.4 \%)$ & $156(25.4 \%)$ & 0.733 \\
\hline
\end{tabular}

* represents $P$ value less than 0.05 between $H$. pylori-positive and $H$. pylorinegative groups.

which this difference was significant $(P=0.011)$. Fasting plasma glucose, in this cohort, also showed a statistically significant difference $(P=0.018)$. According to the TG levels, there was a significant difference in the 2 groups $(1.54 \pm 1.01$ versus $1.40 \pm 0.80, P=0.048)$.

\section{Discussion}

H. pylori, colonized in the human stomach, is an ancient organism that has coevolved with humans for many years [11]. In 1982, Warren and Marshall cultivated the bacterium from mucosal layer of stomach [12]. After three decades, $H$. pylori infection has an influence on approximately $50 \%$ of humans, especially those living in developing countries, which is up to $80 \%$. In this study, the overall infection rate was $31.5 \%$. The infection rate was relatively lower than average infection rates. Since the included human beings were in highly socioeconomic populations, it is widely acknowledged that the incidence of infection is strongly influenced by socioeconomic factors $[13,14]$. Risk factors for $H$. pylori infection are related to living in crowded conditions, living without a reliable supply of clean water, living in a developing country, and living with someone who has a $H$. pylori infection [15].

NAFLD is a common disease that affects $25 \%-30 \%$ of the population in western countries [16]. NAFLD is more often diagnosed among patients with $H$. pylori [17]. According to previous investigation, $H$. pylori infection may be one of the factors that contributes to the pathogenesis of NAFLD. So eradicating $H$. pylori may be significant in the treatment of this disease [18]. However, the pathogenic mechanism of this relationship is unclear. A multitude of previous reports concluded that the infection of $H$. pylori is associated with an altered serum lipid profile. It is also considered a risk factor for atherosclerosis and NAFLD $[2,19]$. H. pylori may lead to liver injury via specific toxins [20]. In addition, invasion of $H$. pylori in the small bowel mucosa might increase gut permeability and facilitate the passage of bacterial endotoxins via the portal vein to the liver [21]. Previous studies have suggested that the association between $H$. pylori infection and NAFLD is controversial. Kim et al. found that patients with 
H. pylori infection were at higher risk of NAFLD development compared to an uninfected individual [2]. Another study of 13,737 Japanese adults reported that $H$. pylori infection is not associated with NAFLD [22]. These findings are in favor with the second result. When patients with NAFLD alone were studied, dividing them based on $H$. pylori infection, gender, and HDL-C had significantly different results. This finding indicates that protective factors for NAFLD were HDL-C and female gender. These results may need to be confirmed in multicenter and prospectively designed studies. The previous studies showed that $H$. pylori infection had a positive association with high LDL-C and low HDL-C $[16,23]$. Similarly, this research agrees with this view.

It is considered that $H$. pylori infection plays a pathophysiologic role in several endocrine disorders. Numerous studies found an association between $H$. pylori infection and diabetes [24, 25]. Nevertheless, there is no agreement among researchers in this regard. A meta-analysis study showed that there was insufficient evidence that $H$. pylori infection worsened glycemic control in patients with diabetes [26]. However, another meta-analysis investigation found that $H$. pylori infection was more frequent in diabetic patients [27]. The mechanism of this relationship is still unclear. In this study, H. pylori infection was etiologically associated with fasting plasma glucose and postprandial blood sugar. Several studies examined the associated between $H$. pylori infection and insulin resistance (IR) [28]. Jeon et al. proposed the possible role of altered gut microbiota in the pathogenesis of insulin resistance and diabetes [29]. These findings were in agreement with the results that $H$. pylori infection was associated with diabetes. However, the results of annual health examination may differ from the patients' true blood glucose levels. Further examination of patients' glycated hemoglobin (HbA1c) was needed to elucidate the association between $H$. pylori infection and diabetes.

Osteoporosis poses a great threat to the elderly population. It is a major cause of bone fracture and morbidity, disability, and mortality in elderly individuals, affecting their families and the health care system [30]. H. pylori infection could lead to chronic local and systemic immune response. The resulting increase in proinflammatory cytokines could affect bone resorption and might increase the risk of osteoporosis system [31]. The risk factors for osteoporosis include cigarette smoking, excessive alcohol consumption, vitamin $\mathrm{D}$ deficiency, and low dietary calcium [32]. Calcium is ionized in acidic conditions and absorbed in the small bowel. Therefore, in either hypochlorhydric or achlorhydric stomachs, calcium absorption is impaired [33]. Conditions causing a decrease in gastric acid secretion status included gastric surgery, use of PPIs, and infection with $H$. pylori $[32,34,35]$. H. pylori infection causes chronic gastritis and induces a permanent inflammatory response that may increase both the gastric and the systemic indexes of inflammation, such as TNF- $\alpha$, IL- 1 , and IL-6 [35]. Shih et al. demonstrated that $H$. pylori infection may be associated with an increased risk of developing osteoporosis in elderly female patients with upper gastrointestinal diseases [36]. Meantime, Figura et al. found that H. pylori infection by strains expressing CagA may be considered a risk factor for osteoporosis in men [35]. In contrast, Fotouk-Kiai et al. revealed that $H$. pylori infection is not associated with BMD changes in the elderly population [37]. Similarly, in this study, it was not revealed that there was a difference of the prevalence of osteoporosis in $H$. pylori-positive and $H$. pylori-negative groups. Maybe it was caused by the relatively low included general population. Moreover, patients with osteoporosis were studied, dividing them based on $H$. pylori infection. Like other subgroups, we also demonstrated that HDL-C was associated with $H$. pylori infection $(P=0.011)$. In addition, according to fasting plasma glucose, there was a significant difference in this subgroup. Jackuliak and Payer revealed that patients with diabetes mellitus have an increased risk of bone fractures [38]. In contrast, Kumar et al. found that the use of oral hypoglycemic agents for a period of three years or more does not significantly affect the BMD in patients with diabetes mellitus [39]. Apparently, this study was in favor with the first conclusion. When we analyzed subgroups of patients with osteoporosis and NAFLD, $H$. pylori infection had a positive association with low HDL-C and high TG.

The evaluation of an association between $H$. pylori infection and causes of chronic renal failure was limited [40]. A recent meta-analysis study demonstrated that end-stage renal disease could be a potential protective factor for $H$. pylori infection [41]. However, $\mathrm{Gu}$ et al. found no evidence of a significant association between $H$. pylori infection and dialysis overall, whereas long-term treatments of more than four years had a significant protective effect [42]. Maybe, patients with long-term dialysis were not required to eradicate $H$. pylori. In this study, we revealed that $H$. pylori infection was in association with uric acid from Table 3.

A few limitations warrant consideration. First, the average age of our study population was $54.0 \pm 9.6$ years. This might be due to the presence of more risk factors for the development of metabolic abnormalities in people who are in this age group. Second, a number of studies have demonstrated that $H$. pylori infection by strains expressing CagA can significantly induce gastric cancer [43]. However, in this study, the included general population were in a physical examination, and the CagA status was not detected. In the further research, we will discuss the role of $H$. pylori infection by strains expressing CagA in metabolic abnormality. Third, other factors, such as being overweight, abdominal circumference, gene, and diet, may affect $H$. pylori inflammation and regulation of glycemic index. Fourth, this study was only a single-center study. Therefore, data collection from young general population and multicenter studies are necessary for future studies.

In summary, it was found that $H$. pylori infection may be an important factor affecting metabolic abnormality and osteoporosis. This indicates that $H$. pylori eradication might play a significant role in reducing the risk of metabolic abnormality and osteoporosis.

\section{Conflicts of Interest}

The authors declare that there is no conflict of interests regarding the publication of this paper. 


\section{Authors' Contributions}

Li-juan Lu and Ning-Bo Hao contributed equally to this work.

\section{Acknowledgments}

The authors would like to thank all patients who participated in this study.

\section{References}

[1] M. D. Zeng, J. G. Fan, L. G. Lu et al., "Guidelines for the diagnosis and treatment of nonalcoholic fatty liver diseases," Journal of Digestive Diseases, vol. 9, no. 2, pp. 108-112, 2008.

[2] T. J. Kim, D. H. Sinn, Y. W. Min et al., "A cohort study on Helicobacter pylori infection associated with nonalcoholic fatty liver disease," Journal of Gastroenterology, vol. 52, no. 11, pp. 1201-1210, 2017.

[3] U. Thalmaier, N. Lehn, K. Pfeffer, M. Stolte, M. Vieth, and W. Schneider-Brachert, "Role of tumor necrosis factor alpha in Helicobacter pylori gastritis in tumor necrosis factor receptor 1-deficient mice," Infection and Immunity, vol. 70, no. 6, pp. 3149-3155, 2002.

[4] B. M. Roesler, E. M. Rabelo-Goncalves, and J. M. Zeitune, "Virulence factors of Helicobacter pylori: a review," Clinical Medicine Insights: Gastroenterology, vol. 7, pp. 9-17, 2014.

[5] M. I. Pereira and J. A. Medeiros, "Role of Helicobacter pylori in gastric mucosa-associated lymphoid tissue lymphomas," World Journal of Gastroenterology, vol. 20, no. 3, pp. 684698, 2014.

[6] E. M. Rabelo-Goncalves, B. M. Roesler, and J. M. Zeitune, "Extragastric manifestations of Helicobacter pylori infection: possible role of bacterium in liver and pancreas diseases," World Journal of Hepatology, vol. 7, no. 30, pp. 2968-2979, 2015.

[7] Y. L. Lin, J. K. Chiang, S. M. Lin, and C. E. Tseng, "Helicobacter pylori infection concomitant with metabolic syndrome further increase risk of colorectal adenomas," World Journal of Gastroenterology, vol. 16, no. 30, pp. 3841-3846, 2010.

[8] G. M. Buzás, "Metabolic consequences of Helicobacter pylori infection and eradication," World Journal of Gastroenterology, vol. 20, no. 18, pp. 5226-5234, 2014.

[9] K. Liu, P. Liu, R. Liu, X. Wu, and M. Cai, "Relationship between serum leptin levels and bone mineral density: a systematic review and meta-analysis," Clinica Chimica Acta, vol. 444, pp. 260-263, 2015.

[10] L. W. Chen, F. P. Chen, C. W. Hsieh, S. F. Kuo, and R. N. Chien, "Analysis of the associations among Helicobacter pylori infection, adiponectin, leptin, and 10-year fracture risk using the fracture risk assessment tool: a cross-sectional community-based study," PLoS One, vol. 12, no. 4, article e0175365, 2017.

[11] Y. Moodley, B. Linz, R. P. Bond et al., "Age of the association between Helicobacter pylori and man," PLoS Pathogens, vol. 8, no. 5, article e1002693, 2012.

[12] N. J. Talley, A. R. Zinsmeister, A. Weaver et al., "Gastric adenocarcinoma and Helicobacter pylori infection," Journal of the National Cancer Institute, vol. 83, no. 23, pp. 17341739, 1991.
[13] P. R. Harris, L. E. Smythies, P. D. Smith, and G. I. Perez-Perez, "Role of childhood infection in the sequelae of $H$. pylori disease," Gut Microbes, vol. 4, no. 6, pp. 426-438, 2013.

[14] M. Epplein, L. B. Signorello, W. Zheng et al., "Race, African ancestry, and Helicobacter pylori infection in a low-income United States population," Cancer Epidemiology, Biomarkers \& Prevention, vol. 20, no. 5, pp. 826-834, 2011.

[15] S. Diaconu, A. Predescu, A. Moldoveanu, C. S. Pop, and C. Fierbinteanu-Braticevici, "Helicobacter pylori infection: old and new," Journal of Medicine and Life, vol. 10, no. 2, pp. 112-117, 2017.

[16] M. Waluga, M. Kukla, M. Zorniak, A. Bacik, and R. Kotulski, "From the stomach to other organs: Helicobacter pylori and the liver," World Journal of Hepatology, vol. 7, no. 18, pp. 2136-2146, 2015.

[17] Z. Dogan, L. Filik, B. Ergul, M. Sarikaya, and E. Akbal, “Association between Helicobacter pylori and liver-to-spleen ratio: a randomized-controlled single-blind study," European Journal of Gastroenterology \& Hepatology, vol. 25, no. 1, pp. 107110, 2013.

[18] S. A. Polyzos, J. Kountouras, A. Papatheodorou et al., "Helicobacter pylori infection in patients with nonalcoholic fatty liver disease," Metabolism, vol. 62, no. 1, pp. 121-126, 2013.

[19] D. Cheng, C. He, H. Ai, Y. Huang, and N. Lu, "The possible role of Helicobacter pylori infection in non-alcoholic fatty liver disease," Frontiers in Microbiology, vol. 8, p. 743, 2017.

[20] N. S. Taylor, J. G. Fox, and L. Yan, "In-vitro hepatotoxic factor in Helicobacter hepaticus, H. pylori and other Helicobacter species," Journal of Medical Microbiology, vol. 42, no. 1, pp. 48-52, 1995.

[21] Y. Fukuda, H. Bamba, M. Okui et al., "Helicobacter pylori infection increases mucosal permeability of the stomach and intestine," Digestion, vol. 63, no. 1, Supplement 1, pp. 93-96, 2001.

[22] K. Okushin, Y. Takahashi, N. Yamamichi et al., "Helicobacter pylori infection is not associated with fatty liver disease including non-alcoholic fatty liver disease: a large-scale crosssectional study in Japan," BMC Gastroenterology, vol. 15, no. 1, p. 25, 2015.

[23] S. Y. Nam, K. H. Ryu, B. J. Park, and S. Park, "Effects of Helicobacter pylori infection and its eradication on lipid profiles and cardiovascular diseases," Helicobacter, vol. 20, no. 2, pp. 125-132, 2015.

[24] K. H. Rhee, J. S. Park, and M. J. Cho, "Helicobacter pylori: bacterial strategy for incipient stage and persistent colonization in human gastric niches," Yonsei Medical Journal, vol. 55, no. 6, pp. 1453-1466, 2014.

[25] A. Takeoka, J. Tayama, H. Yamasaki et al., "Impact of Helicobacter pylori immunoglobulin G levels and atrophic gastritis status on risk of metabolic abnormality," PLoS One, vol. 11, no. 11, article e0166588, 2016.

[26] C. Horikawa, S. Kodama, K. Fujihara et al., "Association of Helicobacter pylori infection with glycemic control in patients with diabetes: a meta-analysis," Journal of Diabetes Research, vol. 2014, Article ID 250620, 7 pages, 2014.

[27] X. Zhou, C. Zhang, J. Wu, and G. Zhang, “Association between Helicobacter pylori infection and diabetes mellitus: a metaanalysis of observational studies," Diabetes Research and Clinical Practice, vol. 99, no. 2, pp. 200-208, 2013.

[28] J. Vafaeimanesh, M. Bagherzadeh, A. Heidari, F. Motii, and M. Parham, "Diabetic patients infected with helicobacter 
pylori have a higher insulin resistance degree," Caspian Journal of Internal Medicine, vol. 5, no. 3, pp. 137-142, 2014.

[29] C. Y. Jeon, M. N. Haan, C. Cheng et al., "Helicobacter pylori infection is associated with an increased rate of diabetes," Diabetes Care, vol. 35, no. 3, pp. 520-525, 2012.

[30] F. J. B. Lötters, J. P. van den Bergh, F. de Vries, and M. P. M. H. Rutten-van Mölken, "Current and future incidence and costs of osteoporosis-related fractures in The Netherlands: combining claims data with BMD measurements," Calcified Tissue International, vol. 98, no. 3, pp. 235-243, 2016.

[31] S. C. Lin, M. Koo, and K. W. Tsai, "Association between Helicobacter pylori infection and risk of osteoporosis in elderly Taiwanese women with upper gastrointestinal diseases: a retrospective patient record review," Gastroenterology Research and Practice, vol. 2014, Article ID 814756, 5 pages, 2014.

[32] H. W. Kim, Y. H. Kim, K. Han et al., "Atrophic gastritis: a related factor for osteoporosis in elderly women," PLoS One, vol. 9, no. 7, article e101852, 2014.

[33] R. R. Recker, "Calcium absorption and achlorhydria," The New England Journal of Medicine, vol. 313, no. 2, pp. 70-73, 1985.

[34] Y. T. Lau and N. N. Ahmed, "Fracture risk and bone mineral density reduction associated with proton pump inhibitors," Pharmacotherapy, vol. 32, no. 1, pp. 67-79, 2012.

[35] N. Figura, L. Gennari, D. Merlotti et al., "Prevalence of Helicobacter pylori infection in male patients with osteoporosis and controls," Digestive Diseases and Sciences, vol. 50, no. 5, pp. 847-852, 2005.

[36] H. M. Shih, T. Y. Hsu, C. Y. Chen et al., "Analysis of patients with Helicobacter pylori infection and the subsequent risk of developing osteoporosis after eradication therapy: a Nationwide population-based cohort study," PLoS One, vol. 11, no. 9, article e0162645, 2016.

[37] M. Fotouk-Kiai, S. R. Hoseini, N. Meftah et al., "Relationship between Helicobacter pylori infection (HP) and bone mineral density (BMD) in elderly people," Caspian Journal of Internal Medicine, vol. 6, no. 2, pp. 62-66, 2015.

[38] P. Jackuliak and J. Payer, "Osteoporosis, fractures, and diabetes," International Journal of Endocrinology, vol. 2014, Article ID 820615, 10 pages, 2014.

[39] B. S. Kumar, A. Ravisankar, A. Mohan et al., "Effect of oral hypoglycaemic agents on bone metabolism in patients with type 2 diabetes mellitus \& occurrence of osteoporosis," The Indian Journal of Medical Research, vol. 141, no. 4, pp. 431437, 2015.

[40] M. Sugimoto and Y. Yamaoka, "Review of Helicobacter pylori infection and chronic renal failure," Therapeutic Apheresis and Dialysis, vol. 15, no. 1, pp. 1-9, 2011.

[41] K. Wijarnpreecha, C. Thongprayoon, P. Nissaisorakarn et al., "Association between Helicobacter pylori and end-stage renal disease: a meta-analysis," World Journal of Gastroenterology, vol. 23, no. 8, pp. 1497-1506, 2017.

[42] M. Gu, S. Xiao, X. Pan, and G. Zhang, "Helicobacter pylori infection in dialysis patients: a meta-analysis," Gastroenterology Research and Practice, vol. 2013, Article ID 785892, 10 pages, 2013.

[43] F. Wang, W. Meng, B. Wang, and L. Qiao, "Helicobacter pylori-induced gastric inflammation and gastric cancer," Cancer Letters, vol. 345, no. 2, pp. 196-202, 2014. 


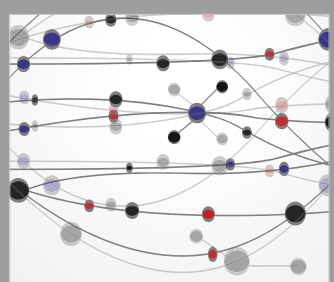

The Scientific World Journal


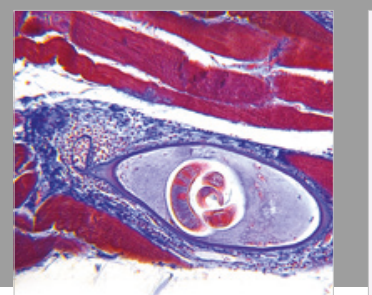

Gastroenterology Research and Practice

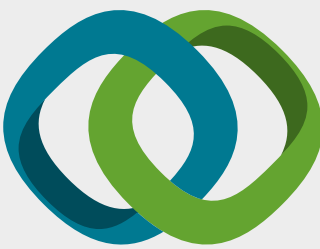

\section{Hindawi}

Submit your manuscripts at

www.hindawi.com
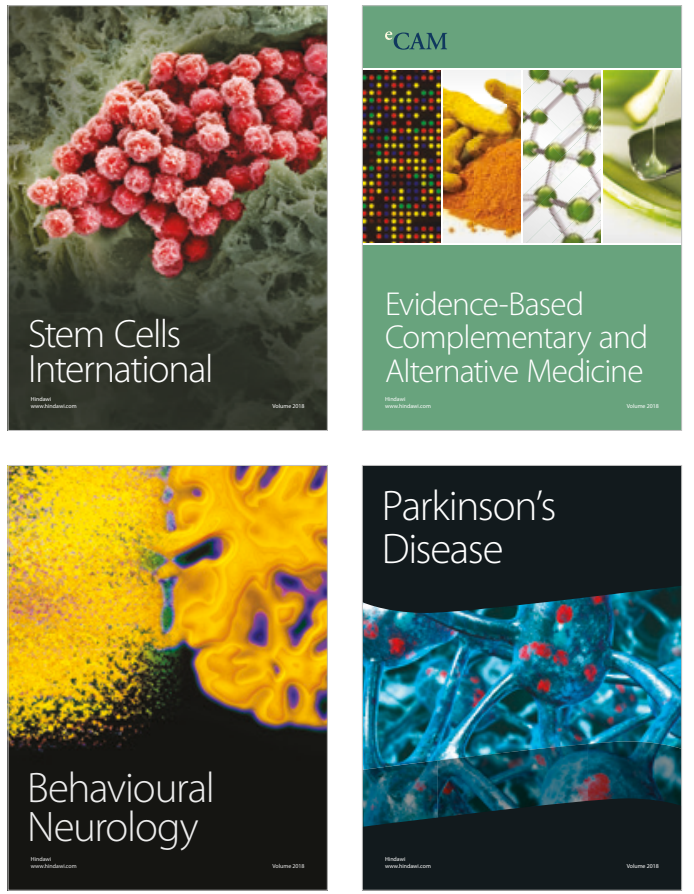

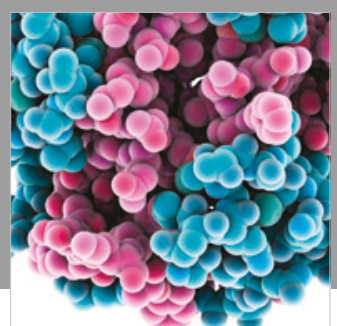

ournal of

Diabetes Research



Disease Markers
